\title{
Modeling Coulombic failure of sea ice with leads
}

Article

Published Version

Wilchinsky, A. V. and Feltham, D. (2011) Modeling Coulombic failure of sea ice with leads. Journal of Geophysical Research - Oceans, 116 (C8). C08040. ISSN 0148-0227 doi: https://doi.org/10.1029/2011JC007071 Available at https://centaur.reading.ac.uk/34581/

It is advisable to refer to the publisher's version if you intend to cite from the work. See Guidance on citing.

Published version at: http://dx.doi.org/10.1029/2011JC007071

To link to this article DOI: http://dx.doi.org/10.1029/2011JC007071

Publisher: American Geophysical Union

All outputs in CentAUR are protected by Intellectual Property Rights law, including copyright law. Copyright and IPR is retained by the creators or other copyright holders. Terms and conditions for use of this material are defined in the End User Agreement.

\section{www.reading.ac.uk/centaur}

\section{CentAUR}

Central Archive at the University of Reading

Reading's research outputs online 


\title{
Modeling Coulombic failure of sea ice with leads
}

\author{
Alexander V. Wilchinsky ${ }^{1}$ and Daniel L. Feltham ${ }^{1}$ \\ Received 23 February 2011; revised 20 April 2011; accepted 2 June 2011; published 30 August 2011.
}

[1] Sea ice failure under low-confinement compression is modeled with a linear Coulombic criterion that can describe either fractural failure or frictional granular yield along slip lines. To study the effect of anisotropy we consider a simplified anisotropic sea ice model where the sea ice thickness depends on orientation. Accommodation of arbitrary deformation requires failure along at least two intersecting slip lines, which are determined by finding two maxima of the yield criterion. Due to the anisotropy these slip lines generally differ from the standard, Coulombic slip lines that are symmetrically positioned around the compression direction, and therefore different tractions along these slip lines give rise to a nonsymmetric stress tensor. We assume that the skewsymmetric part of this tensor is counterbalanced by an additional elastic stress in the sea ice field that suppresses floe spin. We consider the case of two leads initially formed in an isotropic ice cover under compression, and address the question of whether these leads will remain active or new slip lines will form under a rotation of the principal compression direction. Decoupled and coupled models of leads are considered and it is shown that for this particular case they both predict lead reactivation in almost the same way. The coupled model must, however, be used in determining the stress as the decoupled model does not resolve the stress asymmetry properly when failure occurs in one lead and at a new slip line.

Citation: Wilchinsky, A. V., and D. L. Feltham (2011), Modeling Coulombic failure of sea ice with leads, J. Geophys. Res., 116, C08040, doi:10.1029/2011JC007071.

\section{Introduction}

[2] The evolution of the sea ice covers of the polar seas is controlled by atmospheric and oceanic conditions, interactions between the sea ice, atmosphere and ocean, and the mechanical and thermodynamic properties of the ice cover itself. A typical region of the sea ice cover, in the central pack away from coastal and open ocean boundaries, is formed from floes that have lateral dimensions between $100 \mathrm{~m}$ and $5 \mathrm{~km}$, and which are typically several meters thick. During winter, the floes weld together to form larger floe aggregates that form a semicontinuous cover. Under sufficient compressive stresses, typically exerted by the wind, the ice cover can break up and override to form long, narrow piles of rubble above and beneath the ice cover, known as pressure ridges. At more moderate confinement stresses, however, the ice cover breaks up to form long, narrow failure zones, known as leads, whose length typically spans many floe widths. A newly formed lead contains open water, but, unless actively deforming, will quickly freeze over in winter to become a dormant, inactive lead. Since dormant leads contain ice thinner than the surrounding floes, they weaken the ice cover and are often regions of preferential failure. In this paper, we focus our attention on the role of dormant leads in failure

\footnotetext{
${ }^{1}$ National Centre for Earth Observation, Centre for Polar Observation and Modeling, University College London, London, UK.

Copyright 2011 by the American Geophysical Union. 0148-0227/11/2011JC007071
}

under moderate degrees of confinement stress, conditions in which new leads can be expected to form.

[3] Inspection of SAR images like those produced by R. Kwok (Polar Remote Sensing Group) during the SHEBA experiment (Surface Heat Budget of the Arctic Ocean, Perovich et al. [1999]) available at www-radar.jpl.nasa.gov/ rgps/image_files/combine_small.gif with several snapshots given in Figure 1 shows that the most prevalent mode of sea ice deformation occurs through activation of two failure lines with sliding along them. The sliding is usually accompanied by some opening (dilatancy [Tremblay and Mysak, 1997]) as the failure lines are not linear. Where the failure line is due to a new lead being formed, we call this a slip line. If the failure line is created by the reactivation of a dormant lead, we refer to the failure line as an active lead or, more simply, a lead. In some cases opening along failure lines (slip lines or leads) is caused by the invasion of sharp vertices of ice blocks into them. Sometimes activation of failure lines occurs in dormant leads that do not appear to be favorably oriented for failure with respect to the principal compressive direction. We can interpret such failure at nonpreferential failure angles as being due to the presence of thinner ice in these leads, which makes them easier to fail. Figure 1 shows several events of reactivation of dormant leads as well as reactivation of one lead accompanied by formation of a new slip line. Similarly, Schulson [2004] interpreted Landsat-7 images for the Beaufort Sea near the Canadian Archipelago and identified two separate sliding events along faults oriented in different directions that 

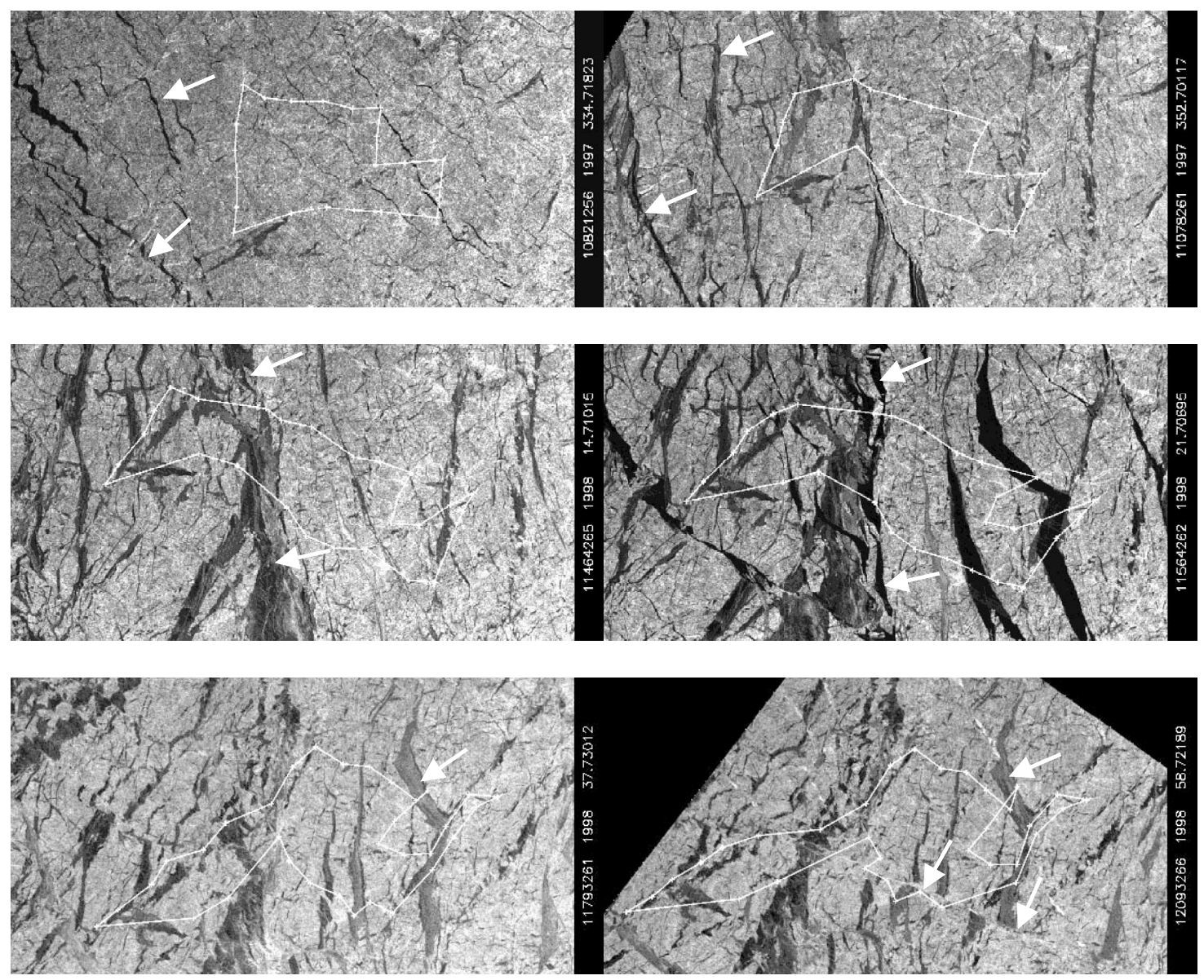

Figure 1. SAR images of three different events of lead reactivation: (left) before reactivation, and (right) after reactivation. The snapshots are taken during SHEBA experiments in the central pack of the Arctic by Polar Remote Sensing Group (http://www-radar.jpl.nasa.gov/rgps/image_files/combine_small.gif). The timing of the images is shown on the right margins. The white arrows point at the reactivated leads. The leads do not undergo any visible deformation for several days before the left snapshot is taken. The two white arrows at the bottom right image that are not present on the corresponding left image show simultaneous formation of a slip line in the isotropic ice.

have presumably been formed by different, consecutive lowconfinement compressive wind stresses. This shows that in some situations new slip lines will form rather than old leads reactivate. Schulson [2004] also compared the Landsat-7 images with SAR images of the same area taken several days earlier and found that shear in one lead changed direction between the dates of these two images, which implies reactivation of the same lead under different stress conditions.

[4] Different approaches have been proposed to model the effect of leads on sea ice rheology; reviews can be found in the work of Hibler [2001a] and Feltham [2008] and there was a special section on small-scale sea ice mechanics in this journal [Kwok and Coon, 2006]. Coon et al. [1998] proposed using an ice thickness distribution, usually used in modeling isotropic sea ice, to describe the ice thickness distribution in leads. They combined the yield curves of isotropic ice and a lead by choosing the weaker between them at any particular stress state to identify a resultant yield curve for sea ice containing a lead. The approach of Hibler and Schulson [2000] was to apply continuity of normal traction at the interfaces of thick ice and thin ice in a lead to find out which lead orientation would require the lowest stress to induce its plastic yield. Schreyer et al. [2006] considered the effect of flaw orientation through decohesive weakening induced by plastic yield. The approach of Wilchinsky and Feltham [2004, 2006a, 2006b] was to develop a continuum model of anisotropic sea ice. The first of these models [Wilchinsky and Feltham, 2004, 2006b] was based on heuristic arguments, while the more recent [Wilchinsky and Feltham, 2006a] assumed that the sea ice cover consisted of diamond shaped blocks based on observations. The shape of such ice blocks is in reality variable and is determined by failure lines during sea ice failure.

[5] Here, we focus on the competition between reactivation of existing leads and the formation of new slip lines in response to a compressive stress state with a low confinement ratio. Our reasons for this are to develop a better understanding of the observed dynamics of small-scale (sub $5-10 \mathrm{~km}$ ) events seen in SAR images, and hence to develop an ability to predict the angle of failure lines. Predicting the angles of failure lines can be useful for studies of regional sea ice dynamics, e.g. sea ice motion through straits, and for 


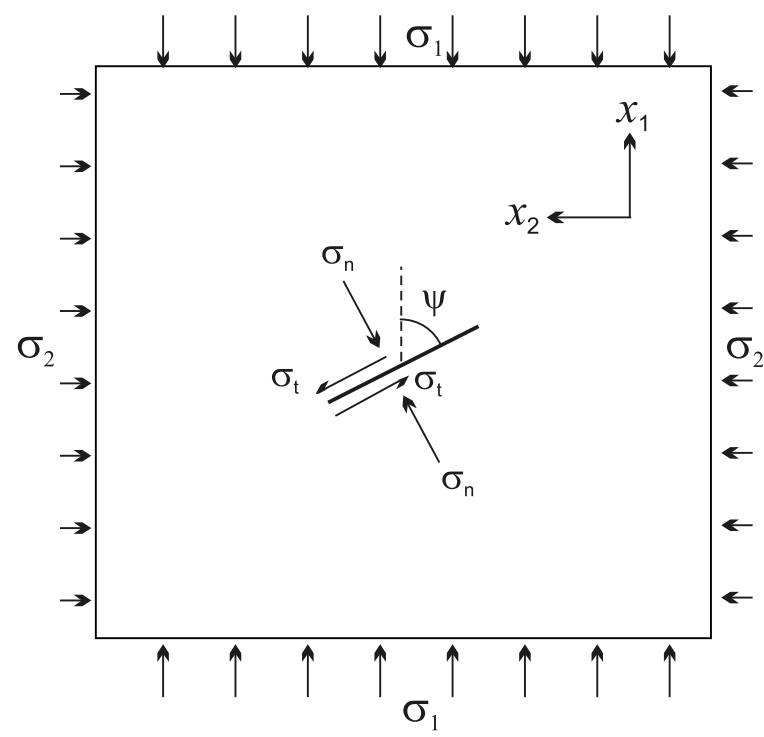

Figure 2. Traction on a line in sea ice. Notation defined in the main text. Reproduced after Ashby and Hallam [1986].

the development of models of large scale sea ice dynamics such as are used for climate modeling. In particular, the rheology of the granular sea ice model [Tremblay and Mysak, 1997] crucially depends on the slip line orientation. Other studies of slip lines in sea ice, with a different focus to this manuscript, include the study of characteristics in a sea ice model with a general plastic failure criterion [Pritchard, 1988] and Coulombic failure [Erlingsson, 1991].

[6] The paper is structured as follows: in section 2 we introduce our basic assumptions and the Coulomb failure criterion, suitably adapted for the thinner ice in leads; in section 3 we use the Coulomb criterion to calculate the orientation and thickness of leads that will fail in preference to the formation of a new slip line, assuming no interaction between failure lines; in section 4 we introduce the concept of a couple stress, which allows us to investigate the effect of failure line interaction, and discuss the sea ice stress state; and finally, in section 5 we summarize our main results and discuss some implications of our work.

\section{The Failure Model}

[7] A reasonably comprehensive description of anisotropic failure of sea ice would require extension of the standard sea ice thickness distribution function into orientational space [Coon et al., 1998] so that the thickness distribution function would show the areal fraction of ice having a particular thickness and orientation at a given location and time. However, based on observation of SAR images we assume that a lead becomes active (fails) with the slip line in the lead parallel to the lead itself so that displacement of the lead sides can occur. In this case lead width does not play any role in lead failure, and we consider a simplified anisotropic sea ice model where each orientation in the sea ice of angle $\psi$ with respect to a coordinate axis in the horizontal plane is described by a particular sea ice/lead thickness: $h(\psi)$, where $h(\psi)=h(\psi+\pi)$ due to symmetry. In the case in which there are a countable number of leads we assert that these leads are embedded in isotropic ice of thickness $\bar{h}$, where $\bar{h}$ denotes the mean thickness averaged over orientation, as the leads do not contribute to the average. The cases in which there is only one or two leads in isotropic ice is similar to those considered by Hibler and Schulson [2000] and Hibler [2001b]. Hibler and Schulson [2000] considered that a lead fails when a Coulombic yield criterion written in terms of stress invariants is satisfied in the lead. This generally allows slip lines in the lead that are not aligned with the lead itself and failure of the ice in the lead is therefore disassociated from sliding along the lead. This approach requires knowledge of the full stress field in the lead, including the longitudinal stress along the lead which cannot be directly determined by the large scale sea ice stress field and is unknown. In contrast, here we shall consider Coulombic slip lines in the failing leads to be aligned with the leads themselves, so that our failure criterion in the lead involves only tractions at the lead-isotropic ice interfaces and is directly determined by the isotropic sea ice stress field. In this case, if $r(\psi)=h(\psi) / \bar{h}$ is the relative lead thickness, then the traction in the lead would be $1 / r$ times higher than the similar traction in the homogenized sea ice of thickness $\bar{h}$. The failure criterion is taken to be

$$
F(\boldsymbol{\sigma})=\sigma_{c},
$$

where $\sigma_{c}$ is cohesion, and the yield function is

$$
F=\left(\left|\sigma_{t}\right|+\mu \sigma_{n}\right) / r,
$$

where $\sigma_{t}, \sigma_{n}$ are shear traction and normal traction in the sea ice respectively (per unit of mean thickness $\bar{h}$ ), and $\mu$ is the friction coefficient. In our later calculations we take $\mu=0.7$ based on the mean value determined by laboratory observation of fault orientations at terminal failure at $-3^{\circ} \mathrm{C}$ and $-10^{\circ} \mathrm{C}$ [Schulson et al., 2006]. While this value of the internal friction coefficient is found on the laboratory scale, Weiss and Schulson, [2007, 2009] argue that it is scale independent from the laboratory scale up to the geophysical scale. In particular, using different temperatures and different techniques of measuring the internal friction coefficient Schulson et al. [2006] found it to be in the range of 0.6 to 1 . At the same time, sea ice stresses recorded during the SHEBA experiment [Richter-Menge et al., 2002] lie within an angle in the stress invariant space determined $\mu=0.9$ as was shown by Weiss and Schulson [2007]. Therefore, here we assume that using the values of the internal friction coefficient found on the laboratory scale in modeling sea ice on the geophysical scale is justified. By comparing laboratory data with SHEBA data, Weiss and Schulson [2007] also show that cohesion decreases by a factor between 15 and 30 when going from the laboratory scale to the geophysical scale, describing the effect of stress concentrators on fault initiation. In particular, shear at zero pressure on the geophysical scale was found to be $40 \mathrm{kPa}$, which gives $\sigma_{c}=$ $48.8 \mathrm{kPa}$ for $\mu=0.7$. This is in contrast to the assumption of zero cohesion adopted by Tremblay and Mysak [1997] in their granular sea ice model. As can be seen from the failure criterion (1)-(2), the presence of cohesion makes Coulombic sea ice failure anisotropic, as otherwise, if cohesion is zero, the effect of the sea ice thickness, $r$, can be eliminated from 


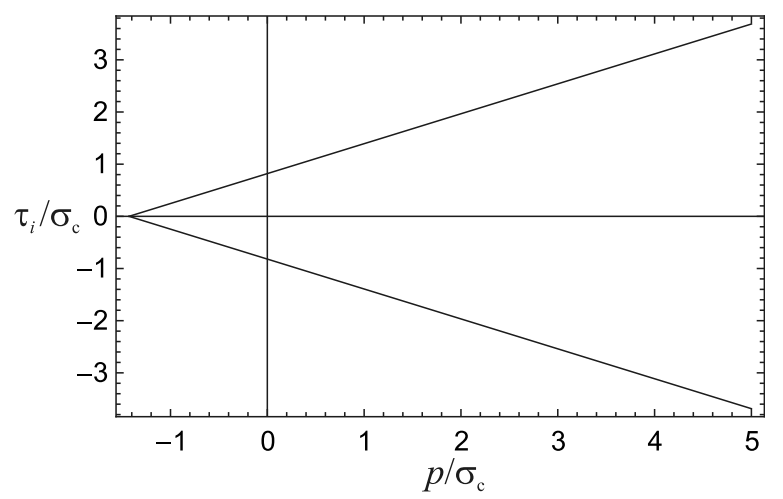

Figure 3. Yield curve for isotropic sea ice failure in stress invariant coordinates.

the failure criterion, and the anisotropy of sea ice thickness will not play any role in Coulombic sea ice failure.

[8] Let us consider a low confinement compression along axis $x_{1}$ so that the coordinate axes coincide with the stress principal axes, and the stress principal components are $\sigma_{1}<$ $\sigma_{2} \leq 0$. Note that compressive stresses are negative, and $\sigma_{1}$ is the most compressive stress while $\sigma_{2}$ is the least compressive stress. Similar to Ashby and Hallam [1986], we consider a positive angle $\psi$ in the clockwise rotation direction from the compression direction $x_{1}$ (Figure 2). For a positive lead angle $\psi>0$ the tractions are

$$
\left|\sigma_{t}\right|=\tau \sin 2 \psi, \quad \sigma_{n}=\tau \cos 2 \psi-p,
$$

where the stress tensor invariants, pressure and shear (per unit thickness of $\bar{h}$ ) are

$$
p=-\left(\sigma_{1}+\sigma_{2}\right) / 2>0, \quad \tau=\left(\sigma_{2}-\sigma_{1}\right) / 2>0 .
$$

For a negative angle $\psi$, the plus sign at $\sin 2 \psi$ should be changed to a minus here and in the subsequent formulas. In terms of pressure and shear, the failure criterion takes the form

$$
F(p, \tau, \psi, r)=\frac{1}{r(\psi)}[\tau \sin 2 \psi+\mu(\tau \cos 2 \psi-p)]=\sigma_{c} .
$$

[9] In order to find a direction at which the slip lines would form, one has to find a maximum of $F$ with regard to angle $\psi$ as this is where the yield criterion would be attained first under progressive loading. For smooth functions, this can be done by determining $\psi$ from $\partial F(p, \tau, \psi, r) / \partial \psi=0$. For an orientation dependent $r$ the found angle generally depends on both $p$ and $\tau$. However, since failure at any particular angle requires satisfaction of (1), this can be used to associate $p$ and $\tau$ in finding a maximum of $F$, so that the found angle will depend on only one of $\tau$ or $p$. We will choose $p$ to be arbitrary, and the failure criterion (5) then determines the corresponding shear stress

$$
\tau=\frac{r \sigma_{c}+\mu p}{\sin 2 \psi+\mu \cos 2 \psi}
$$

In isotropic ice $r$ is constant $(r=1)$, and the critical flaw angle in the isotropic ice determined by a maximum $F$ is not dependent on the stress and is

$$
\psi_{c}=\frac{1}{2} \arctan (1 / \mu) \text {. }
$$

For $\mu=0.7$, the critical flaw angle is $27.5^{\circ}$. Similarly, if a number of leads intersect the isotropic ice, but still the isotropic ice fails rather than the leads, then it would fail at the same critical angle $\psi_{c}$ as this is where the yield function is maximum. If this happens, satisfaction of the yield criterion (1) at the slip line will determine the yield shear stress of isotropic sea ice failure $\tau_{i}$ as a function of pressure as in (6), given $\psi=\psi_{c}$ and $r=1$,

$$
\tau_{i}=\frac{\sigma_{c}+\mu p}{\left(1+\mu^{2}\right)^{1 / 2}},
$$

which is presented in Figure 3.

[10] The found dependency between the shear and the pressure (6) implies that Coulombic failure can occur only at a stress confinement ratio $R_{c}=\sigma_{2} / \sigma_{1}$ lower than a critical value $R_{c}^{*}$. Given that, from (4), $p=-\sigma_{1}\left(1+R_{c}\right) / 2$ and $\tau=$ $\sigma_{1}\left(R_{c}-1\right) / 2$ the yield criterion (5) can be rewritten as

$$
\frac{\sigma_{1}}{r(\psi)}\left[\left(R_{c}-1\right)(\sin 2 \psi+\mu \cos 2 \psi)+\mu\left(R_{c}+1\right)\right]=\sigma_{c} .
$$

If the expression in the brackets is zero then the left hand side cannot attain the value of cohesion so that no Coloumbic failure would occur. Setting the term in brackets to zero determines the upper limit on the confinement ratio at an arbitrary failure angle $\psi$ as

$$
R_{c}^{*}=\frac{\sin 2 \psi+\mu \cos 2 \psi-\mu}{\sin 2 \psi+\mu \cos 2 \psi+\mu}
$$

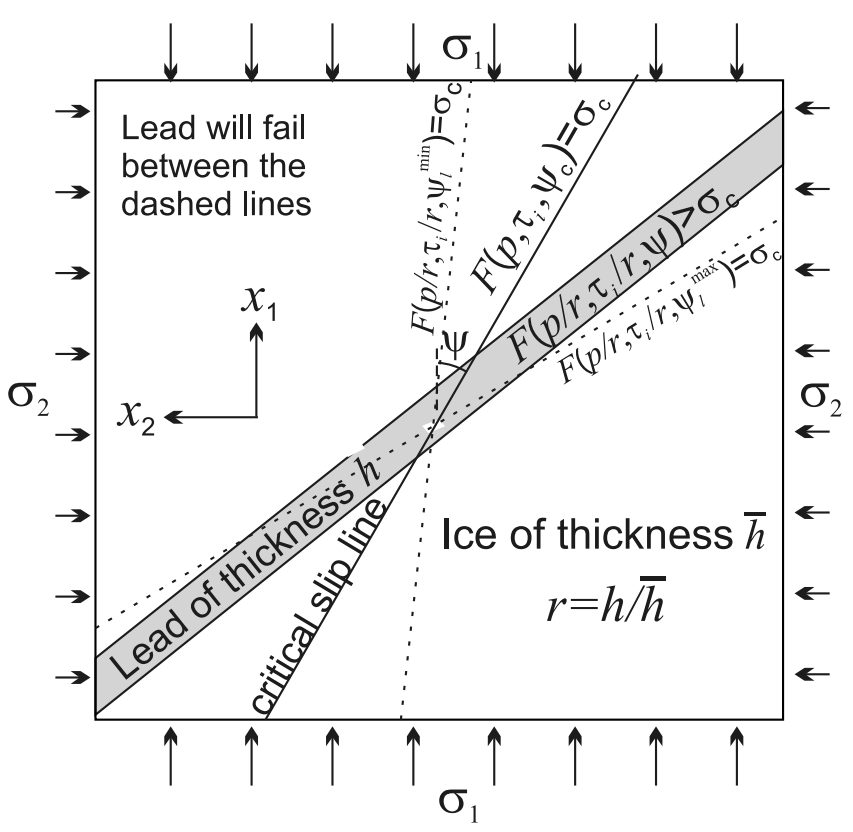

Figure 4. Failure of a single lead versus failure in the isotropic ice at the critical angle. 

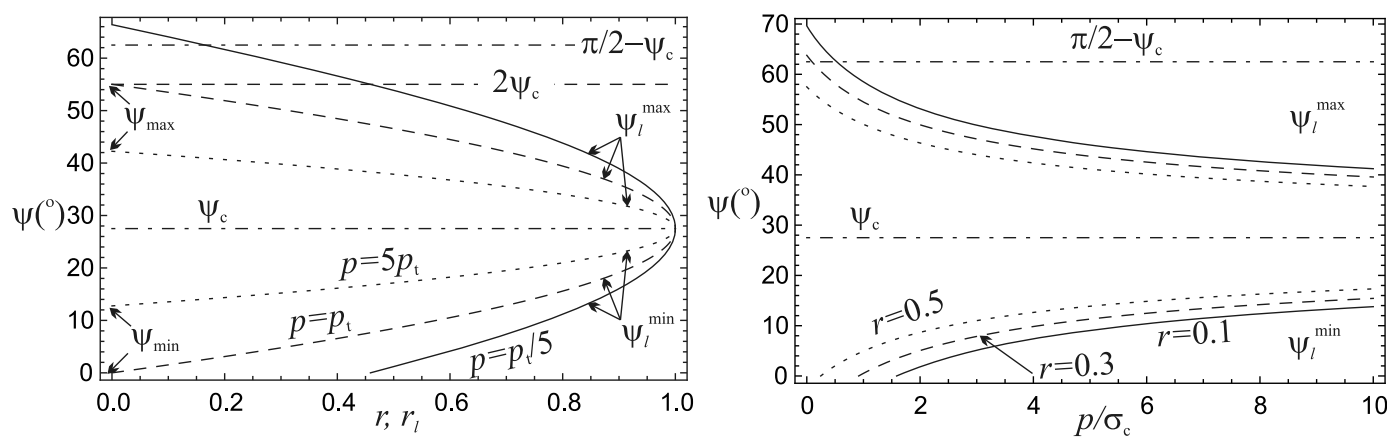

Figure 5. The limiting angles $\psi_{l}^{\min }$ and $\psi_{l}^{\max }$ describing the angle range of lead failure (left) versus the relative lead thickness $r$ for three fixed pressure magnitudes $p=p_{t} / 5$ (compression under transverse tension), $p_{t}$ (uniaxial compression) and $5 p_{t}$ (compression under confinement) for the decoupled model; and (right) versus the pressure for three fixed values of $r=0.1,0.3$ and 0.5 . The curve of the limiting lead thickness $r_{l}(\psi)$ is visualized by considering the abscissa as the ordinate and vice versa in the left image. Here $\psi_{\min }$ and $\psi_{\max }$ are determined by intersection of the curves with the axis $r_{l}=0$ left and right of the critical angle $\psi_{c}$, respectively.

which is independent of the thickness. In the case of isotropic ice with failure at the critical angles the critical confinement ratio takes the following form [Schulson, 2001]

$$
R_{c}^{*}=\frac{\left(1+\mu^{2}\right)^{1 / 2}-\mu}{\left(1+\mu^{2}\right)^{1 / 2}+\mu} .
$$

\section{A Decoupled Lead Model}

\subsection{A Single Lead in Uniform Ice}

[11] Let us now consider only one lead in isotropic ice (Figure 4) under confinement. Failure will either occur in the lead or in the isotropic ice at the critical angle. In order to identify which scenario is to occur, the value of the yield function in the lead and in the isotropic ice at the critical angle should be compared. In particular, if we consider the stress field determined by failure in the isotropic ice, $F\left(p, \tau_{i}\right.$, $\left.\psi_{c}, 1\right)=\sigma_{c}$, then from the failure criterion (2) it is clear that $F\left(p, \tau_{i}, \psi_{c}, r\right)>\sigma_{c}$ for $r<1$. Therefore if there is a lead lying at an angle close to $\psi_{c}$ then the lead would fail rather than the isotropic ice. As the lead rotates away from the critical angle in both directions the value of the yield function decreases until it reaches its isotropic ice failure magnitude $\sigma_{c}$ at the limits $\psi_{l}^{\min }<\psi_{c}<\psi_{l}^{\max }$, and both the lead and isotropic ice can fail simultaneously. Equating the yield functions for the lead ice to cohesion $\sigma_{c}, F\left(p, \tau_{i}, \psi, r\right)=\sigma_{c}$ at shear stress $\tau_{i}$ determined by isotropic ice failure (equations (5) and (8)), determines the preferential lead angle failure range

$$
\begin{gathered}
\psi_{l}^{\min / \max }=\arccos \left[\frac{f_{1} \pm f_{2}}{2\left(1+\mu^{2}\right)}\right]^{1 / 2}, \\
f_{1}=1+\mu^{2}(1+s)+\mu k, \\
f_{2}=\left[1+\mu^{2}\left(1-s^{2}\right)-k^{2}-2 \mu k s\right]^{1 / 2}, \\
s=p / \tau_{i}, \quad k=r \sigma_{c} / \tau_{i}, \quad \tau_{i}=\left(\sigma_{c}+\mu p\right) /\left(1+\mu^{2}\right)^{1 / 2} .
\end{gathered}
$$

A plus sign in (12) determines the lower boundary $\psi^{\min }$, while the minus sign determines the upper boundary $\psi^{\text {max }}$. Whether a lead aligned with the compression direction can ever fail is determined by the critical pressure

$$
p_{t}=\sigma_{c} /\left[\mu-\left(1+\mu^{2}\right)^{1 / 2}\right]
$$

which is found by requiring $\psi_{l}^{\min }(p, r=0)=0$, and separates compression under confinement (higher pressures) from compression under transverse tension (lower pressures). For $\mu=0.7, p_{t}=1.92 \sigma_{c}$.

[12] The lead failure angle range (12) is plotted in Figure 5 versus the relative lead thickness $r$ for fixed pressure magnitudes times $p_{t}$, and versus the normalized pressure $p / \sigma_{c}$ for fixed lead thicknesses. It can be seen that reducing the pressure widens the lead failure angle range since, as can be seen from (2), a lower pressure means a higher yield function magnitude so that a larger deviation from the critical angle is required to compensate for this increase in yield function. Similarly, increasing the lead thickness leads to narrowing of the lead failure angle range as this involves reducing the stress in the lead and consequently reducing the yield function magnitude, so that only a smaller deviation from the critical angle is allowed.

\subsection{Regimes of Lead Failure as the Compression Direction Changes: Two Decoupled Leads Initially at Critical Angles}

[13] In order for sea ice to undergo a general deformation, failure should occur along (at least) two failure lines. Section 3.1 describes the range of angles around the direction of the largest principal stress axis for a single lead to fail in preference to the isotropic ice. If the lead lies out of this range, the isotropic ice would fail at the standard critical angle (7). If two leads lie nonsymmetrically around the compression direction, or their thicknesses are different, then failure in one lead would influence how the other lead forms, and a coupled lead model must be considered, as is done in section 4 . Here, however, we consider a decoupled lead failure model where the results of section 3.1 are applied to each lead separately. In homogeneous sea ice two leads will form at the standard critical angles $\pm \psi_{c}$ around the 


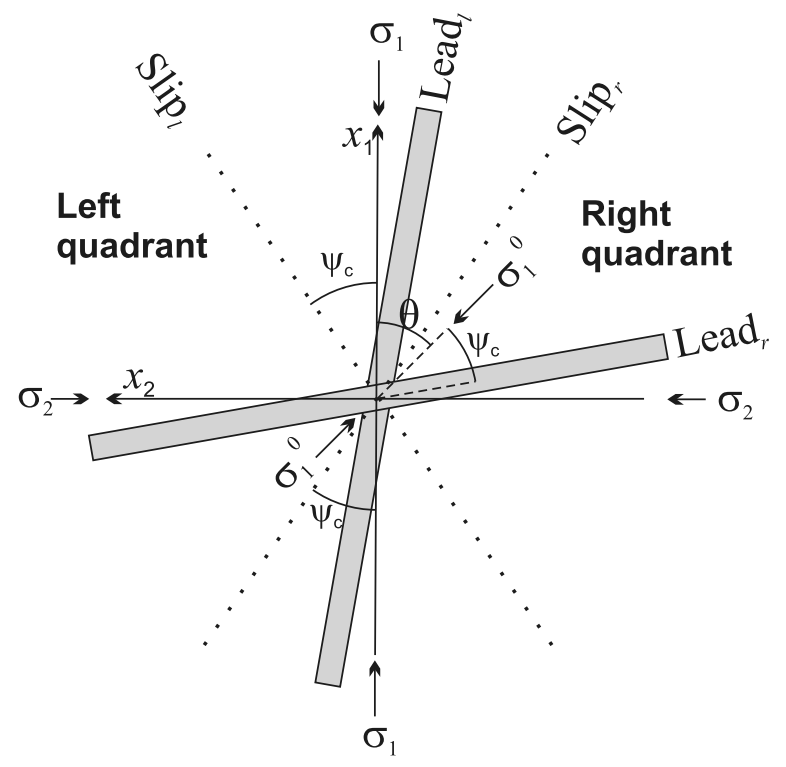

Figure 6. Initial and possible new lead orientation as the most compressive stress direction rotates counterclockwise. Here $\sigma_{1}^{0}$ is the initial most compressive stress leading to the symmetric initial formation of $\operatorname{Lead}_{l}$ and $\operatorname{Lead}_{r}$ either side of its direction.

compression direction. If a next compression event occurs with the principal compressive axis at a different angle, both initial leads may fail, or only one of them will fail accompanied by a new slip line formed in the isotropic ice, or the leads will remain inactive, while two new slip lines form in the isotropic ice. Using a discrete element model, Wilchinsky et al. [2011] studied how a $90^{\circ}$ wind direction change affects the distribution of active leads. Since the model included Coulombic failure at floe edges, the active lead distribution was determined by an interplay between the nonavailability of leads at a particular angle (negative effect) and the higher shear stress intensity in them in comparison to the isotropic ice (positive effect). Due to boundary effects in the model's square domain, Wilchinsky et al. [2011] restricted their analysis to a change in wind direction of $90^{\circ}$. Here we use the analytical approach described above to study how an arbitrary rotation of the compression direction from its initial direction, around which two leads at critical angles have formed, would affect reactivation of the leads (Figure 6). Due to the symmetry of the geometry, considering rotation angles of up to $\pi / 2$ is sufficient.

[14] Solution (12) described the angle range for preferential lead failure having a particular relative thickness $r$. While this solution is convenient if the lead thickness is fixed, we are interested in analyzing a general situation of arbitrary lead thickness to see how this affects lead reactivation and isotropic ice failure. If we assume that the failure criterion (5) holds at any given angle under the stress conditions determined by the isotropic ice failure, we can find such a limiting lead thickness $r_{l}$ that satisfies the failure criterion at this angle, so that if a lead has a lower thickness than $r_{l}$ then it will fail. Rearranging (5) with $\tau=\tau_{i}$ (equation (8)) accordingly yields

$$
r_{l}(\psi, p)=\frac{1}{\sigma_{c}}\left[\tau_{i}(\sin 2 \psi+\mu \cos 2 \psi)-\mu p\right] .
$$

The curve $r_{l}(\psi)$ is visualized by considering the abscissa as the ordinate and vice versa in the left panel of Figure 5. If $r_{l}$ of two leads is compared, then the lead that has a larger $r_{l}$ would have a larger factor multiplying the shear stress in the yield function (5) as the pressure term is the same, and therefore between these two leads that with a larger $r_{l}$ will preferentially fail. We can rewrite $r_{l}$ in terms of the deviation angle from the standard critical angle, $\psi_{d}=\psi-\psi_{c}$, to be

$$
r_{l}\left(\psi_{d}, p\right)=\frac{1}{\sigma_{c}}\left[\left(\sigma_{c}+\mu p\right) \cos 2 \psi_{d}-\mu p\right],
$$

which shows that the function is symmetric around the critical flaw angle where it attains its maximum, which can also be seen from its plots in Figure 5. Changing the pressure shifts and stretches $r_{l}$ vertically.

[15] The geometry of the problem we consider is given in Figure 6, where by $\operatorname{Lead}_{l}$ and $\operatorname{Lead}_{r}$ we denote two initial leads inclined left and right at critical angles $\psi_{c}$ around the initial compression direction, while by $\mathrm{Slip}_{l}$ and $\mathrm{Slip}_{r}$ we denote two possible new slip lines in the isotropic ice inclined left and right around the new compression direction. Without the initial leads, failure would always occur along these slip lines. The angle of rotation of the compression direction is denoted by $\theta$, and the problem geometry is completely described by the lead positions in the left and right quadrants shown in Figure 6. Initially, at $\theta=0$, the leads lie symmetrically around $x_{1}$ in both the left and the right quadrant. As the most compressive direction rotates away from its original position that caused the initial formation of leads $\operatorname{Lead}_{l}$ and $\operatorname{Lead}_{r}$, these leads can lie either in different quadrants or both in the right quadrant. Since the number of the initial leads and their position in each quadrant associated with each new possible slip line varies depending on rotation angle $\theta$, one has to consider competition of failure along each of the new possible slip lines $\mathrm{Slip}_{l}$ and Slip $_{r}$ versus the initial leads separately. This is possible as we consider a decoupled lead model.

[16] Putting $r=0$ in (12) determines the failure angle range for an open lead, [ $\left.\psi_{\min }, \psi_{\max }\right]$, within which any lead with $r \leq r_{l}(\psi, p)$ will fail rather than the isotropic ice at the critical angle:

$$
\psi_{\min / \max }=\arccos \left\{\frac{1+\mu^{2}[1+s] \pm\left[1+\mu^{2}\left(1-s^{2}\right)\right]^{1 / 2}}{2\left(1+\mu^{2}\right)}\right\}^{1 / 2} .
$$

At uniaxial compression pressure $p=p_{t}$, given by (14) ensuring $\psi_{\min }=0$, a lead aligned with the new compression direction would fail only if it is open as $r_{l}\left(\psi=0, p_{t}\right)=0$. Moreover, due to the symmetry of $r_{l}$ around the critical angle $\psi_{c}$, considering $p=p_{t}$ also determines $\psi_{\max }-\psi_{\min }=$ $2 \psi_{c}$ (Figure 5, left), that is at the pressure $p=p_{t}$ two leads initially lying at critical angles can fail both simultaneously only if they are open and lie at angles 0 and $2 \psi_{c}$ relative to the new compression direction. For a pressure higher than $p_{t}, p>p_{t}$, we have $\psi_{\min }>0$ and $\psi_{\max }-\psi_{\min }<2 \psi_{c}$ (Figure 5), that is only one initial lead can be within the failure range. For a pressure lower than $p_{t}, p<p_{t}, \psi_{\text {min }}$ remains zero (Figure 5), and, since $r_{l}(0, p)>0$ in this case, a lead lying along the compression direction can preferentially fail if it 


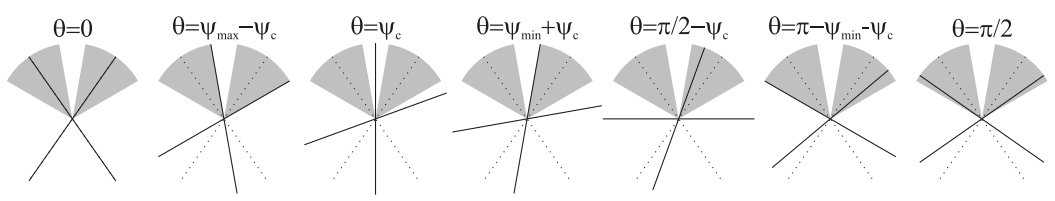

Figure 7. Different thresholds of rotation of compression direction away from its original position (indicated by a clockwise rotation of the black leads away from the isotropic critical failure lines shown by dotted lines). The gray shaded fans describe the maximum lead failure range (for $r=0$ ), shown only in the upper semiplane. For illustrative purposes the critical angle here is taken as $35^{\circ}$, so that $\operatorname{Lead}_{r}$ reenters the failure range in the left quadrant, while the failure range does not include the compression direction, as is the case for $\mu=0.7$.

is thinner than $r_{l}$. Since now $\psi_{\max }-\psi_{\min }>2 \psi_{c}$ (Figure 5), two initial leads can be within the failure range, however, the structure of (15), where weakening of pressure $p$ only raises and stretches the curve vertically, implies that $r_{l}$ at these two leads are equal only when the leftmost lead, $\operatorname{Lead}_{l}$, is along the compressive direction. Otherwise, $r_{l}$ at Lead d $_{l}$ would be larger than at $\operatorname{Lead}_{r}$ and, given the leads have the same thickness, $\operatorname{Lead}_{l}$ would preferentially fail.
[17] As the compression direction rotates away from its original position by up to $\pi / 2$, the leads pass through different sea ice failure regimes (Figure 7). Figure 8 indicates the presence of active leads and slip lines depending on the rotation angle and the lead thickness. The shaded regions show the domains in $\{\theta, r\}$ space in which failure of the initial leads $\operatorname{Lead}_{l}$ and $\operatorname{Lead}_{r}$ occurs, versus the potential new slip lines $\mathrm{Slip}_{l}$ and $\mathrm{Slip}_{r}$ lying at critical angles around the
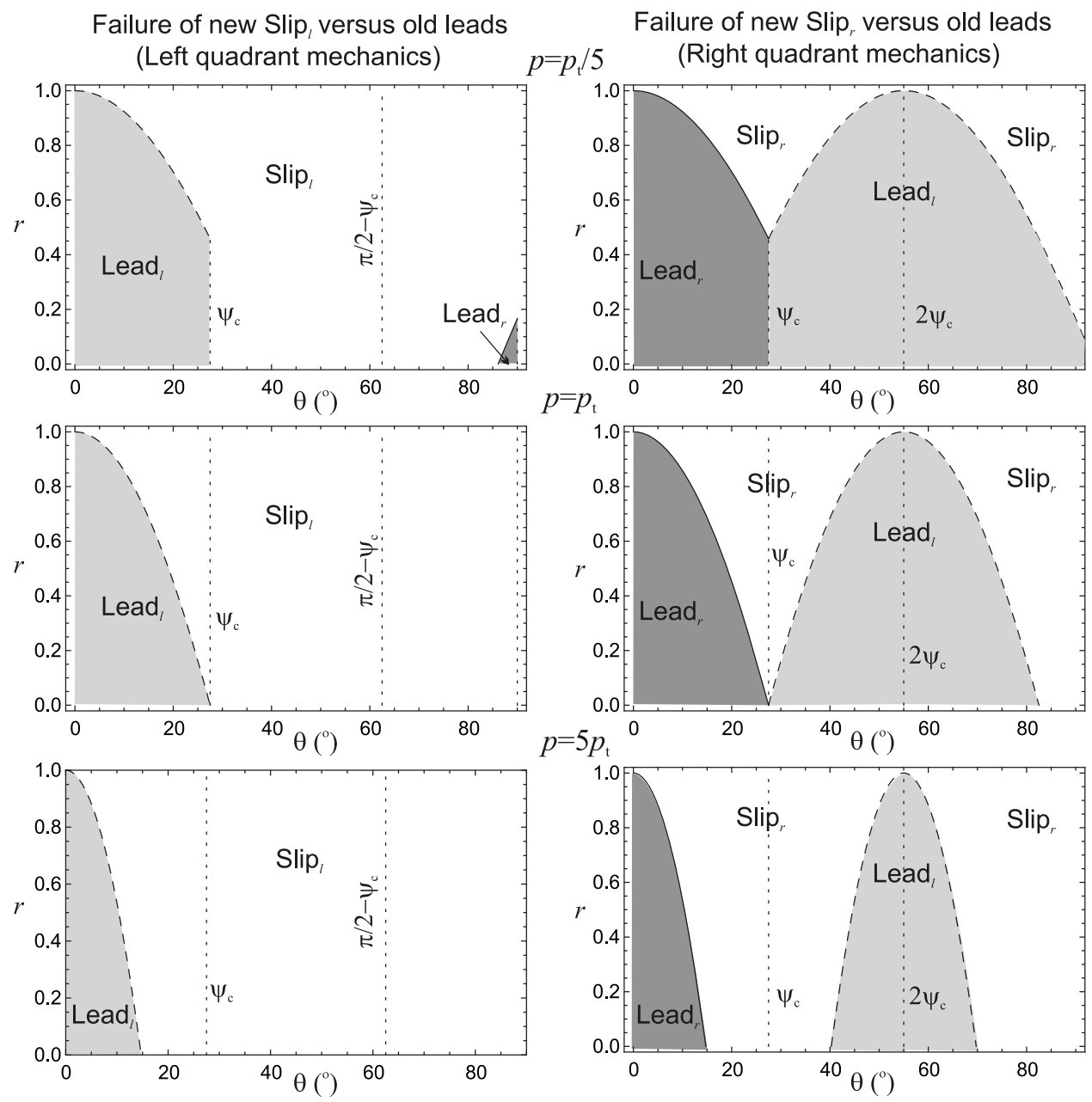

Figure 8. The domain $\{\theta, r\}$ of values of compression direction rotation angle $\theta$ counterclockwise and of the lead thickness $r$ in which failure of $\operatorname{Lead}_{l}$ and Lead ${ }_{r}$ occurs versus slip line formation at standard critical angles $\mathrm{Slip}_{l}$ and $\mathrm{Slip}_{r}$ for the decoupled model. The domain boundaries are delineated by $r_{l}(\theta, p)$. The solid line and darker shades denote the domains of failure of $\operatorname{Lead}_{r}$, while the dashed line and lighter shades denote domains of failure of $\operatorname{Lead}_{l}$. 
new compression direction. The lead failure domains are delineated by $r_{l}(\theta, p)$. The cases of pressure being lower, equal, and higher than $p_{t}$ are shown separately. Initially the leads lie at the critical angles around the compression direction, where the yield function attains its maximum, and, since the leads are thinner than the isotropic ice, they will fail. As the rotation angle increases the lead angles deviate from the critical angles until they leave the lead failure angle range if $p>p_{t}$ for any lead thickness or when $r>r_{l}(0, p)$ otherwise. The leads leave the failure range simultaneously due to the symmetry of the yield function around the critical angles. If $p<p_{t}$ for any lead thickness or when $r<r_{l}(0, p)$ then the lead failure angle range extends to the compression direction, and $\mathrm{Lead}_{l}$ leaves the left quadrant and enters the right quadrant before $\operatorname{Lead}_{r}$ leaves the lead failure angle range. When $\operatorname{Lead}_{l}$ lies along the compression direction the yield function at both leads has the same magnitude, however as the rotation angle increases Lead $_{l}$ begins approaching the critical slip line in the right quadrant so its yield function increases, while Lead $_{r}$ rotates further away from the critical slip line and its yield function decreases. As a consequence, failure in the right quadrant now occurs in Lead $_{l}$ while Lead $r$ becomes inactive. Since there is no lead left in the left quadrant, failure there occurs in a slip line. For high angles of rotation Lead $_{r}$ leaves the right quadrant and enters the left quadrant. In some situations $\operatorname{Lead}_{r}$ can enter the lead failure angle range in the left quadrant, if it is sufficiently wide. The final position of $\operatorname{Lead}_{r}$ given by $\psi=$ $\psi_{c}-\pi / 2$ is the closest to the failure range in the left quadrant $\left[-\psi_{\max },-\psi_{\min }\right]$. The pressure $p_{r}$ that ensures that the final lead position coincides with the maximum failure range limit, $\psi_{\max }(p)=\pi / 2-\psi_{c}$, given by

$$
p_{r}=\frac{f \sigma_{c}}{f \mu+\left(1+\mu^{2}\right)^{1 / 2}}, \quad f=\cos 2 \psi_{c}-\sin 2 \psi_{c} / \mu,
$$

separates cases where $\operatorname{Lead}_{r}$ reenters the failure range and becomes active again in the left quadrant preventing formation of $\operatorname{Slip}_{l}\left(p<p_{r}\right)$ from those in which it does not and failure in the left quadrant occurs in $\operatorname{Slip}_{l}\left(p>p_{r}\right)$.

[18] Due to its simplicity the developed model of decoupled leads clearly demonstrates the interplay between failure of old leads and formation of new slip lines. However, in cases where a mixed failure takes place, that is simultaneously in a lead and along a new slip line, the same pressure gives rise to different shear stresses (6) determined by the failure criterion. As a consequence, if, similar to Wilchinsky and Feltham [2006a], one would write the stress tensor in terms of the tractions at these two failure lines, then the found pressure would generally be different from pressure $p$, and the stress tensor will be nonsymmetric. Such an inconsistency of the decoupled model is due to the initial assumption that the stress tensor determined through anisotropic failure is symmetric. In section 4 we consider a coupled model by considering a nonsymmetric stress tensor arising through anisotropic failure.

\section{A Coupled Lead Model}

[19] As mentioned above, the decoupled lead model gives rise to a number of inconsistencies when the associated stress is considered. The issue arises as we start from the assumption that the stress tensor is symmetric (as is the case in standard Coulombic failure of isotropic ice), while, in anisotropic sea ice, the difference in tractions along nonsymmetric slip lines/active leads results in a stress, which we shall call the couple stress (following Cowin [1974]), that would, unopposed, result in a spin of the floes. The existence of the couple stress is caused by the simplicity of our model, where a homogeneous stress field is considered and the slip lines are infinite. In reality this spin will be suppressed by the surrounding sea ice field that imposes an additional stress that counteracts the floe spin. One would expect this additional stress to be mainly concentrated at floe vertices. Similar to Wilchinsky and Feltham [2006a] we assume that the additional stress from the sea ice field can be taken into account by considering only the symmetric part of the stress tensor arising from the tractions at the slip lines. This is analogous to assuming that the additional stress arising through floe spin suppression by the surrounding sea ice field is described by a skewsymmetric stress tensor that does not contribute to work as the plastic deformation spin tensor is zero. However, while the whole sea ice stress in this case is symmetric its constituent that determines tractions in leads is generally nonsymmetric, which must be assumed in considering a coupled model of leads.

[20] A nonsymmetric stress tensor can be represented through a sum of the standard symmetric part $\sigma$, described by two principal values $\sigma_{1}<\sigma_{2}$, where $\sigma_{1}$ is associated with the most compressive principal stress direction, and a skewsymmetric part, whose form does not depend on the coordinate system, and is described by only one parameter representing the couple stress magnitude, $\tau_{s}$ :

$$
\boldsymbol{\sigma}^{*}=\boldsymbol{\sigma}+\tau_{s}\left(\begin{array}{cc}
0 & 1 \\
-1 & 0
\end{array}\right)
$$

In this case in its principal axes the stress field is described not only by the usual invariants of the symmetric part of the stress tensor, $p$ and $\tau$, but also by the couple stress $\tau_{s}$. As in the case of our decoupled lead model, here we consider a coordinate system that is aligned with the principal axes of the symmetric part of the stress tensor. We consider the pressure as a free parameter, while the shear stress and the couple stress will be determined by sea ice failure. The presence of the skewsymmetric stress does not affect normal traction on any surface, while its shear traction contribution is $\tau_{s}$. After taking into account that shear traction is taken positive in the yield function, the failure criterion (5) becomes

$$
\begin{aligned}
& F\left(p, \tau, \tau_{s}, \psi, r\right) \\
& =\left\{\begin{array}{l}
\frac{1}{r}\left[\tau \sin 2 \psi-\tau_{s}+\mu(\tau \cos 2 \psi-p)\right]=\sigma_{c}, \quad \psi>0 \\
\frac{1}{r}\left[\tau_{s}-\tau \sin 2 \psi+\mu(\tau \cos 2 \psi-p)\right]=\sigma_{c}, \quad \psi<0
\end{array}\right.
\end{aligned}
$$

In order to accommodate an arbitrary deformation, two failure lines are necessary. Their directions are determined by the maxima of the yield function. In an isotropic case the maxima are attained simultaneously at the standard critical angles $\pm \psi_{c}$. In an anisotropic case, where the ice thickness depends on orientation, the yield function maxima can have different magnitudes. Therefore, the failure would not happen simul- 
taneously, but rather the weakest direction will fail first. The couple stress arises due to the difference in tractions on two nonsymmetric failure lines. Therefore, finding the first failure direction in subyield sea ice does not require consideration of a nonzero couple stress. For a countable number of leads we can determine whether a lead will fail first (become active), rather than a pair of new slip lines form in the isotropic ice at standard critical angles, by checking if a lead lies within the range given by (12). If no leads lie within this range, then failure will occur along two new slip lines in the isotropic ice at angles $\pm \psi_{c}$. If only one lead lies within this range, then this will be the first lead to fail. If there are several leads lying within this range, then one must compare the yield functions at these leads. Given a fixed pressure, failure of a lead determines a corresponding shear stress given by (6). Comparing two leads lying at angles $\psi_{1}$ and $\psi_{2}$, the one that will fail first will have a smaller shear, and if they have the same thickness then the lead lying at $\psi_{1}$ will fail first if

$$
\frac{\tau_{1}}{\tau_{2}}=\frac{\left|\sin 2 \psi_{2}\right|+\mu \cos 2 \psi_{2}}{\left|\sin 2 \psi_{1}\right|+\mu \cos 2 \psi_{1}}<1,
$$

and vice versa.

[21] After the first failure lead and its angle $\psi_{1}$ have been determined, satisfaction of the failure criterion there relates the couple stress $\tau_{s}$ to the shear $\tau$ for a given pressure $p$ (rearrangement of (20) where $\psi=\psi_{1}$ ):

$$
\tau_{s}=\left\{\begin{array}{ll}
\tau\left(\sin 2 \psi_{1}+\mu \cos 2 \psi_{1}\right)-\left(\mu p+r_{1} \sigma_{c}\right), & \psi_{1}>0 \\
\tau\left(\sin 2 \psi_{1}-\mu \cos 2 \psi_{1}\right)+\left(\mu p+r_{1} \sigma_{c}\right), & \psi_{1}<0
\end{array} .\right.
$$

Note that the factor multiplying the shear is positive for positive angles and negative for negative angles.

[22] In order for a second failure line to form, the shear stress must increase beyond the value ensuring the first failure. This increase in shear is accompanied by an increase in the couple stress magnitude $\tau_{s}$ that ensures that the failure criterion stays satisfied at the first failure line. Inspection of the yield criterion (20) reveals that at the first failure the factor multiplying the maximum shear $\tau$ is positive as the couple stress is zero, and the pressure cannot be lower than minus cohesion. From (22) we see that increasing the shear $\tau$ increases the couple stress magnitude, with $\tau_{s}$ becoming positive for $\psi_{1}>0$ and negative for $\psi_{1}<0$. For a constant $r$ this change in $\tau_{s}$ tends to decrease the yield function (20) at angles lying in the same quadrant as the first failure line, and to increase the yield function at angles lying in the opposite quadrant. This means, for example, that if the second failure line is a new isotropic ice slip line (e.g. since there are no other leads), then it would be the one that lies in the opposite quadrant relative to the first failure line.

[23] Generally speaking, the higher magnitude of the yield function in the quadrant opposite to that with the first lead for a constant $r$ does not preclude a situation when a second failure occurs in another lead lying in the same quadrant as the first lead due to the effect of a variable $r$. However, suppose that two leads lie in the same quadrant at angles $\psi_{1}$ and $\psi_{2}$ (which we take to be positive for concreteness) then the failure criterion (20) can be written for each of the two leads and the couple stress algebraically eliminated to yield

$$
\tau=\frac{\left(r_{1}-r_{2}\right) \sigma_{c}}{\sin 2 \psi_{1}-\sin 2 \psi_{2}+\mu\left(\cos 2 \psi_{1}-\cos 2 \psi_{2}\right)} .
$$

This equation would need to be satisfied if both leads lie in the same quadrant. Suppose, for example, that both leads had the same thickness, $r_{1}=r_{2}$, then this shows, since $\tau \neq 0$, that the failure criterion cannot be satisfied simultaneously at two leads lying in the same quadrant and therefore the second failure should occur in the quadrant opposite to the first failure quadrant.

[24] For two failure angles $\psi_{1}>0$ and $\psi_{2}<0$, one of which (say, $\psi_{1}$ ) describes the first failure line, writing the failure criteria (20) along each line and eliminating the couple stress yields

$$
\begin{aligned}
& \tau=\frac{\left(r_{1}+r_{2}\right) \sigma_{c}+2 \mu p}{D\left(\psi_{1}, \psi_{2}\right)}, \\
& D\left(\psi_{1}, \psi_{2}\right)=\sin 2 \psi_{1}-\sin 2 \psi_{2}+\mu\left(\cos 2 \psi_{1}+\cos 2 \psi_{2}\right) .
\end{aligned}
$$

The second failure line is where the shear is the minimum over all orientations and the potential isotropic ice slip line $\left(r=1, \psi_{2}=-\psi_{c}\right)$. Suppose that the second failure occurs in a lead with thickness $r_{2}=r_{1}=r$, equating the shear stress to that determined by the second failure occurring along a standard isotropic ice slip line at $-\psi_{c}$ (i.e. using equation (24) to set $\left.\tau\left(r, \psi_{2}\right)=\tau\left(1,-\psi_{c}\right)\right)$ determines a critical lead thickness $r=r_{s}$, below which the second failure will occur in the lead rather than the isotropic ice

$$
r_{s}=\frac{2 \mu\left(p / \sigma_{c}\right)\left[D\left(\psi_{1}, \psi_{c}\right)-D\left(\psi_{1}, \psi_{2}\right)\right]-D\left(\psi_{1}, \psi_{2}\right)}{D\left(\psi_{1}, \psi_{2}\right)-2 D\left(\psi_{1}, \psi_{c}\right)} .
$$

\subsection{Regimes of Lead Failure as the Compression Direction Changes: Two Coupled Leads Initially at Critical Angles}

[25] Here we again consider a simplified case of two leads of the same relative thickness $r$ initially lying at the critical angles $\pm \psi_{c}$ around an initial compression direction. The compression direction rotates counterclockwise by an arbitrary angle of up to $90^{\circ}$. As was discussed above, first we have to find where failure would occur first. Exactly as in the decoupled model, leads lying within the lead failure angle range given by (12) or whose thickness is smaller than the limiting thickness $r_{l}$ given by (15) will preferentially fail rather than new slip lines forming at the critical angles. If there are no such leads, then two new slip lines will form simultaneously in the isotropic ice. If there is only one lead lying within the range, then this will be the lead that will fail first. In our model there are two leads separated by an angle of $2 \psi_{c}$. This makes this situation special. As was discussed earlier, (16) shows that the yield function is symmetric about the critical angles separated by a $2 \psi_{c}$ angle. As the compression direction rotates, the initial leads also rotate away from the critical directions into the regions of smaller and larger angles than the critical angles that could be identified 


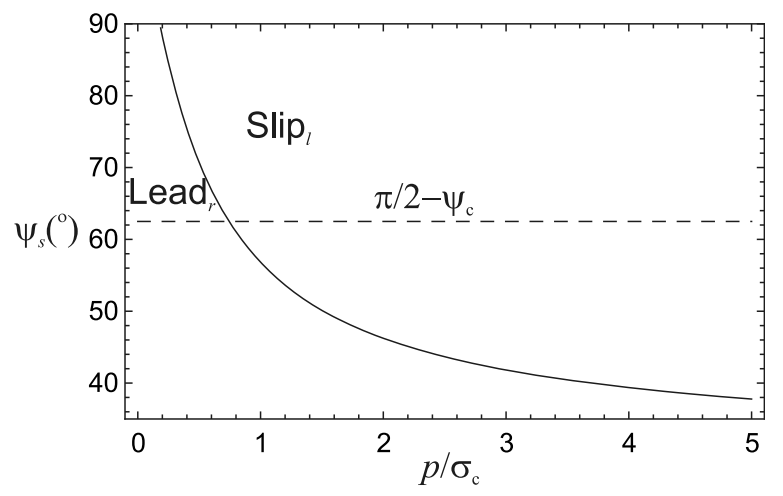

Figure 9. The absolute values of $\psi_{s}$ showing the lower angle limit of $\mathrm{Lead}_{r}$ reactivation in the left quadrant, for the two leads scenario described in the text. The angle of Lead $_{r}$ can only lie between $\pi / 2-\psi_{c}$, its final direction, and $\pi / 2$, where the lead reenters the left quadrant.

as the white areas in the right quadrant in Figure 7. Due to the symmetry of the lead angles around the initial compression direction and the leads being of the same thickness, the magnitude of the yield function $F$ at the leads changes by the same amount and remains equal in the two leads. This means that the initial leads will remain in the lead failure angle range together and will leave it simultaneously (Figure 7, second pictogram). Until this happens, or until one of the leads reaches the compression direction and crosses it into another quadrant, the failure criteria in the both leads are satisfied simultaneously, and they would fail at once. Because of this the active leads are found exactly as in our decoupled model: at small angles of rotation of the compression direction, the initial leads both lie in the failure range. As can be inferred from Figure 8 , for $p>p_{t}$ as the rotation angle increases they leave the lead failure range simultaneously, and then the two new slip lines form in the isotropic ice at critical angles. For $p<p_{t}$ the compression direction itself lies within the lead failure range when $r<$ $r_{l}(0, p)$, and $\operatorname{Lead}_{l}$ crosses this direction while still being active. As $\operatorname{Lead}_{l}$ crosses the compression direction, there are no initial leads remaining in the left quadrant, so that failure will occur in the left quadrant in the isotropic ice along the critical direction. In the right quadrant, there are now two leads with $\operatorname{Lead}_{l}$ having a larger limiting thickness $r_{l}$ than $\operatorname{Lead}_{r}$, so that $\operatorname{Lead}_{l}$ will preferentially fail given it lies within the failure range, otherwise failure would occur in the isotropic ice in the right quadrant also. As the compression direction rotates even further Lead $_{r}$ reenters the left quadrant from below, when the compression direction rotation angle reaches $\pi / 2-\psi_{c}$. As the compression direction rotates further up to the final $\pi / 2$ angle, this lead rotates closer to the critical flaw position in the left quadrant, and likelihood of its failure increases. Failure in the left quadrant failure will now either occur in Lead $_{r}$ or along the isotropic ice slip line $\operatorname{Slip}_{l}$. $\operatorname{Lead}_{r}$ will preferentially fail if its thickness is less than the critical thickness given by (25). If Lead ${ }_{r}$ lies within the range where $r_{s}<0$, then failure in this quadrant would always occur in the isotropic ice in the standard way as by definition $r_{s} \geq 0$ and leads with $r_{s}<0$ are impossible.
Expressing the angle of $\operatorname{Lead}_{r}$ in the left quadrant through the angle of $\operatorname{Lead}_{l}$ in the right quadrant $\psi_{r}=\psi_{l}+2 \psi_{c}-\pi$ and assuming that the first failure occurs in $\operatorname{Lead}_{l}$ we can set $r_{s}$ to zero in equation (25) with $\psi_{1}=\psi_{l}$ and $\psi_{2}=\psi_{r}$, and solve for $\psi_{r}$. This will determine the lower limit angle of unconditional isotropic ice failure in the left quadrant whose absolute value we denote as $\psi_{s}$ :

$$
\begin{gathered}
\psi_{s}=\arctan \left[-\frac{\xi_{2}+\left(\xi_{1}^{2}+\xi_{2}^{2}-\xi_{3}^{2}\right)^{1 / 2}}{\xi_{1}+\xi_{3}}\right], \\
\xi_{1}=-2 \mu^{2} \frac{p}{\sigma_{c}}-\frac{2\left(\mu^{2}-1\right) \mu}{1+\mu^{2}}, \quad \xi_{2}=2 \mu \frac{p}{\sigma_{c}}-\frac{2\left(\mu^{2}-1\right)}{1+\mu^{2}}, \\
\xi_{3}=-2 \mu \frac{p}{\sigma_{c}}\left(1+\mu^{2}\right)^{1 / 2} .
\end{gathered}
$$

The above formula gives the positive magnitude of an angle $\psi_{s}$ such that if $\operatorname{Lead}_{r}$ lies at an angle greater than $\psi_{s}$ from the compressive axis (measured counterclockwise) then failure will occur in the isotropic ice. Note that in the left quadrant $\operatorname{Lead}_{r}$ always lies within the range $\left[\pi / 2-\psi_{c}, \pi / 2\right]$ and $\operatorname{Lead}_{r}$ can fail in the left quadrant only when the pressure magnitude is less than the cohesion, as shown in Figure 9.

[26] In the derivation of equation (26) we assumed that the first failure occurs in a lead in the right quadrant. Generally speaking, for $p>p_{t}$ and high rotational angles the failure in the right quadrant will occur in the isotropic ice since $\psi_{\max }<$ $\pi / 2-\psi_{c}$ in this case. In the decoupled model this would mean that $\operatorname{Lead}_{r}$ is out of the failure range as its angle magnitude cannot be larger than that of $\operatorname{Lead}_{l}$ in the right quadrant. However, in the coupled model $\psi_{s}$ differs from $\psi_{\text {max }}$, therefore if $\psi_{s}>\psi_{\text {max }}$ then $\operatorname{Lead}_{r}$ in the left quadrant could be within the failure range, while $\operatorname{Lead}_{l}$ could be out of the failure range. However, in the decoupled model pressure $p_{r}$ in (18), that determines the threshold for $\operatorname{Lead}_{r}$ to be able to become active again in the left quadrant, was found through equating $\psi_{\max }=\pi / 2-\psi_{c}$. At this pressure the maximum limit for the failure range coincides with the minimum possible angle magnitude of $\operatorname{Lead}_{r}$ in the left quadrant, which is $\pi / 2-\psi_{c}$. However, at this angle the situation is symmetric around the compression axis as both leads lie at the same angle and have the same thickness. Therefore, the decoupled model is adequate in this case, so that $\psi_{s}\left(p_{r}\right)=\psi_{\max }\left(p_{r}\right)$ and this pressure is also the strongest pressure when reactivation of $\operatorname{Lead}_{r}$ in the left quadrant is possible in the coupled model. We conclude, then, that when two leads of the same thickness lying at the critical angles are considered sea ice failure regimes can be adequately determined by the decoupled lead model apart from when $\operatorname{Lead}_{r}$ becomes active again in the left quadrant, which requires $p<p_{r}$. For $\mu=0.7$, from (18) we can find that $p_{r}=$ $0.74 \sigma_{c}$.

\subsection{Regimes of Lead Failure of Two Coupled Leads Initially at Critical Angles: Anisotropic Stress Correction}

[27] The symmetric part of the stress due to Coulombic failure in the coupled lead model is completely determined, given the principal axes, by the linear relationship between shear stress and pressure given by equation (19). Rewriting 


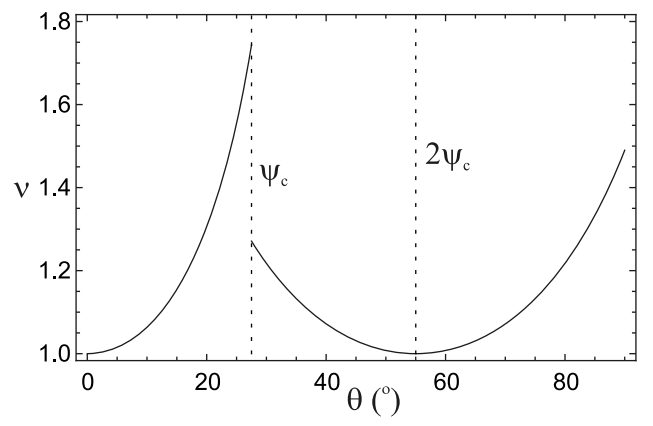

Figure 10. The anisotropic failure correction factor $\nu$ versus the compression direction rotation angle.

(19) into the form of the standard Coulombic failure criterion in isotropic ice (8) yields

$$
\tau=\frac{\eta \sigma_{c}+\nu \mu p}{\left(1+\mu^{2}\right)^{1 / 2}}
$$

$$
\begin{aligned}
\nu & =\frac{2\left(1+\mu^{2}\right)^{1 / 2}}{\sin 2 \psi_{1}-\sin 2 \psi_{2}+\mu\left(\cos 2 \psi_{1}+\cos 2 \psi_{2}\right)}, \\
\eta & =\left(r_{1}+r_{2}\right) \nu / 2,
\end{aligned}
$$

where $\nu$ and $\eta$ describe the correction to the isotropic case formula, and $\psi_{1}>0$ and $\psi_{2}<0$ are the angles between the active failure lines and compression direction, while $r_{1}$ and $r_{2}$ are their thicknesses $\left(r_{1}=r_{2}=1\right.$ if failure occurs in the isotropic ice). The anisotropic correction factor $\nu$ describes the main effect of lead activation on the rheology. As can seen from Figure 8, depending on the lead thickness and the pressure magnitude, for $\theta<\psi_{c}$ failure occurs either in both leads or in the isotropic ice. Since the scenario when $\operatorname{Lead}_{r}$ becomes active again in the left quadrant requires a relatively small pressure describing compression under transverse tension, we discard this possibility here. For $\theta>\psi_{c}$, failure either occurs in the isotropic ice only (along $\operatorname{Slip}_{l}$ and $\mathrm{Slip}_{r}$ ) or along $\mathrm{Slip}_{l}$ and $\mathrm{Lead}_{l}$. Whenever at least one lead fails, which depends on the lead thickness and the rotation angle, anisotropic correction will occur as described by $\nu$. The factor $\nu$, shown in Figure 10 for the whole possible range of rotation angles, increases with the rotation angle until $\operatorname{Lead}_{l}$ crosses the compression direction. The increase in $\nu$ occurs because, for a given pressure, failure along the isotropic slip lines ensures the minimum shear stress so deviation from these lines leads to a higher shear stress for the same pressure. After $\operatorname{Lead}_{l}$ enters the right quadrant, it starts approaching the isotropic slip line $\operatorname{Slip}_{r}$ so that $\nu$ decreases and reaches its minimum at $\theta=2 \psi_{c}$ where $\operatorname{Lead}_{l}$ coincides with $\mathrm{Slip}_{r}$. Generally, the presence of leads can almost double of the factor multiplying the pressure in the equation for shear stress (28). It should, however, be noted that the pressure ridging threshold would limit the possible pressure and depends on the lead thickness. Thus, despite a lower $\nu$, shear stress in ice with no leads can reach higher values than in ice with leads due to possible higher pressures. In particular, if the force necessary to ridge a $1 \mathrm{~m}$ long ridge of thickness $h$ is $\Phi(h)$, then the corresponding normal traction in a lead or a slip line during ridging will be $\Phi(h) / \bar{h}$, which cannot be exceeded. The form of the function $\Phi$ differs in different models. Rothrock [1975] related ice strength in convergence to the change in potential energy involved in forming a pressure ridge (and keel). Discrete element simulations by Hopkins [1998] carried out for a particular set of material parameters reveal that a pressure ridge forms by growth of the sail until a buckling threshold is reached. The force necessary to increase sail height is determined by pushing a train of blocks over the sail surface, which, for the adopted values of material parameters, is equal to $7300 h^{3 / 2} L^{1 / 2} \mathrm{~N} \mathrm{~m}^{-1}$, where $L$ is the length of lead ice pushed into the ridge. The next phase starts when this force reaches the buckling force, $95400 h^{3 / 2} \mathrm{~N} \mathrm{~m}^{-1}$, which happens always at the same $L=L_{f}=107.7 \mathrm{~m}$. Although the ridging force varies depending on the stage of ridge formation, it is noteworthy that $\Phi$ is proportional to $h^{3 / 2}$.

[28] At the active leads, or isotropic ice slip lines, positioned at $\psi_{1}$ and $\psi_{2}$ the traction is given by (3), where the shear stress is given by (24). Taking into account that the normal traction is negative, we can write

$$
p-\frac{\left(r_{1}+r_{2}\right) \sigma_{c}+2 \mu p}{D\left(\psi_{1}, \psi_{2}\right)} \cos 2 \psi \leq \frac{1}{\bar{h}} \Phi(h),
$$

which determines the upper limit on the pressure

$$
p \leq \min _{\psi=\psi_{1}, \psi_{2}} \frac{D\left(\psi_{1}, \psi_{2}\right) \Phi(h) / \bar{h}+\left(r_{1}+r_{2}\right) \sigma_{c} \cos 2 \psi}{D\left(\psi_{1}, \psi_{2}\right)-2 \mu \cos 2 \psi} .
$$

The maximum pressure is plotted in Figure 11 as a function of the rotation of the compression direction for $\Phi=90 h^{3 / 2} \mathrm{kN} \mathrm{m}^{-1}$, $\bar{h}=3 \mathrm{~m}$ and $\sigma_{c}=48.8 \mathrm{kPa}$ [Weiss and Schulson, 2007]. The maximum pressure depends on the orientation and thickness at the two failure lines. At the same time the failure lines depend on the pressure. Therefore in calculations the pressure was gradually increased from zero determining the corresponding failure regime (the active leads or slip lines) until it reached the maximum pressure given by (31) determined by this failure regime. At small angles the failure occurs in the leads and the maximum pressure is low. When $\operatorname{Lead}_{l}$ approaches the compression direction (at $\theta=$ $27.5^{\circ}$ ), the maximum pressure is determined by failure along the standard slip lines in the isotropic ice, so that the maximum pressure reaches it maximum, which is orientation independent as the slip lines form at the same angles with

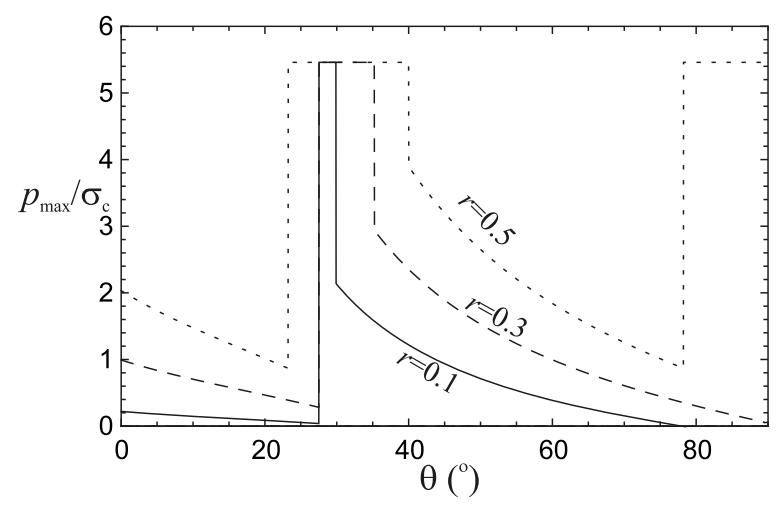

Figure 11. The maximum pressure for different lead thicknesses versus the compression direction rotation angle. 
regard to the compression direction. For higher rotational angles, failure in the right quadrant at the maximum pressure again occurs in a lead, which reduces the maximum pressure. At angles close to $90^{\circ}$ failure occurs along two slip lines in the isotropic ice for the higher lead thicknesses so that the maximum pressure increases again, and in the two leads for the lower lead thickness accompanied by reduction of the maximum pressure.

\section{Summary and Concluding Remarks}

[29] Motivated by high resolution SAR deformation imagery [Kwok, 2001] and numerical studies [Wilchinsky et al., 2010, 2011], we have addressed the question of how existing leads, not generally favorably oriented for failure, affects the formation of new slip lines in sea ice under compressive stress. We have adopted the Coulomb failure criterion, which can model both fracture failure of ice [Schulson, 2001] and granular yield [Tremblay and Mysak, 1997], as the criterion for the formation of new slip lines or the reactivation of leads. The crucial feature of the adopted yield criterion is a nonzero cohesion that determines an anisotropic Coulombic response of sea ice with an orientation-dependent thickness. Without cohesion, even if the ice thickness distribution is anisotropic, Coulombic failure would occur in an isotropic way, similar to Tremblay and Mysak [1997], since the Coulomb failure criterion is independent of ice thickness in this case. A general in-plane failure of the sea ice cover requires the presence of at least two failure lines, therefore, as a particular case our focus was on considering two leads embedded in an isotropic, thick ice cover. First, a decoupled lead model was considered in which the viability of lead failure versus the formation of a new isotropic ice slip line is assessed on both sides of the principal compression direction independently. Then, we considered a coupled lead model in which the formation of a lead affects, through a couple stress, the formation of the second lead or isotropic ice slip line. It was found that, apart from a scenario of a low compressive pressure describing compression under transverse tension, the decoupled model is adequate for predicting the failure of leads versus the formation of new isotropic ice slip lines. However, correct determination of the sea ice stress requires a coupled lead model and a nonsymmetric stress tensor, which is determined by satisfaction of the Coulomb criterion at failure lines that are generally nonsymmetric around the compression direction. The skewsymmetric part of the sea ice stress tensor is a result of a difference in traction on the nonsymmetric slip lines giving rise to a couple stress, and while it must be taken into account in determining sea ice failure regimes, it is assumed to be counterbalanced by the surrounding ice field, whose effect is not accounted for in this simple model directly.

[30] Our calculations show that the viability of lead failure depends on lead thickness and direction relative to the standard isotropic ice slip lines around the compression direction, where the yield function attains its maximum for a given ice thickness. The closer the leads are to the slip lines and the thinner they are, the more likely the leads are to fail. As the leads rotate away from the isotropic slip line positions, they can leave the lead failure range, in which case they do not fail and the isotropic ice fails along the standard slip lines. For large angles of deviation of the leads from the slip line direction, both leads may occupy the same quadrant made by the stress principal axes, in which case failure occurs along one slip line in the isotropic ice in the quadrant with no leads, and one of the leads in the other quadrant (unless both leads are out of the lead failure range, in which case failure occurs along an isotropic slip line in this quadrant also). For even larger angles of deviation, one of the leads turns past the confinement axis (so there are again leads in each quadrant) and this lead may become active again, although this requires a relatively small compressive pressure, less than the cohesion.

[31] Understanding of slip line formation and lead activation has implications for studies in which lead direction is important, for example the decomposition of wind drag across ice and leads, snow transport (deposition of snow into open leads), and for the representation of mechanical anisotropy in sea ice models and the calculation of sea ice stress. Modeling studies [e.g., Wilchinsky et al., 2006], reveal the importance of sliding forces in determining the momentum balance and sea ice thickness distribution. The granular model of sea ice [Tremblay and Mysak, 1997] directly relates sea ice deformation and stress to the slip line orientation. By determining the conditions under which new slip lines will form instead of the activation of existing leads, we can account for the evolution of anisotropy due to these failure lines. If the sea ice anisotropy is modeled through the means of continuum mechanics, e.g. through evolution of a structure tensor [Wilchinsky and Feltham, $2006 \mathrm{~b}$, then formation of new failure lines in compression will represent the source terms for increased anisotropy. The sea ice stress, since it is calculated from the tractions at slip lines/leads, depends upon the level and direction of anisotropy.

\section{References}

Ashby, M. F., and S. D. Hallam (1986), The failure of brittle solids containing small cracks under compressive stress states, Acta Metall., 34, 497-510.

Coon, M., G. S. Knoke, and D. C. Echert (1998), The architecture of anisotropic elastic-plastic sea ice mechanics constitutive law, J. Geophys. Res., 103, 21,915-21,925.

Cowin, S. C. (1974), The theory of polar fluids, Adv. Appl. Mech., 14, 279-347, doi:10.1016/S0065-2156(08)70034-6.

Erlingsson, B. (1991), The propagation of characteristics in sea-ice deformation fields, Ann. Glaciol., 815, 73-80.

Feltham, D. L. (2008), Sea ice rheology, Ann. Rev. Fluid Mech., 40, 91-112, doi:10.1146/annurev.fluid.40.111406.102151.

Hibler, W. D., III (2001a), Sea ice fracturing on the large scale, Eng. Fract. Mech., 68, 2013-2043, doi:10.1016/S0013-7944(01)00035-2.

Hibler, W. D., III (2001b), Modeling the formation and evolution of oriented fractures in sea ice, Ann. Glaciol., 33, 157-164.

Hibler, W. D., III, and E. M. Schulson (2000), On modeling the anisotropic failure and flow of flawed sea ice, J. Geophys. Res., 105, 17,105-17,120, doi:10.1029/2000JC900045.

Hopkins, M. A. (1998), Four stages of pressure ridging, J. Geophys. Res., 103, 21,883-21,891.

Kwok, R. (2001), Deformation of the Arctic Ocean sea ice cover: November 1996 through April 1997, in IUTAM Symposium on Scaling Laws in Ice Mechanics and Ice Dynamics, Solid Mechanics and Its Applications, vol. 94, edited by J. P. Dempsey and H. H. Shen, pp. 315-323, Springer, New York.

Kwok, R., and M. D. Coon (2006), Introduction to special section: SmallScale Sea Ice Kinematics and Dynamics, J. Geophys. Res., 111, C11S21, doi:10.1029/2006JC003877.

Perovich, D. K., et al. (1999), Year on ice gives climate insights, Eos Trans. $A G U, 80,485-486$.

Pritchard, R. S. (1988), Mathematical characteristics of sea ice dynamics models, J. Geophys. Res., 93, 15,609-15,618. 
Richter-Menge, J. A., S. L. McNutt, J. E. Overland, and R. Kwok (2002), Relating arctic pack ice stress and deformation under winter conditions, J. Geophys. Res., 107(C10), 8040, doi:10.1029/2000JC000477.

Rothrock, D. A. (1975), The energetics of the plastic deformation of pack ice by ridging, J. Geophys. Res., 80, 4514-4519.

Schreyer, H. L., D. L. Sulsky, L. B. Munday, M. D. Coon, and R. Kwok (2006), Elastic-decohesive constitutive model for sea ice, J. Geophys. Res., 111, C11S26, doi:10.1029/2005JC003334.

Schulson, E. M. (2001), Brittle failure of ice, Eng. Fract. Mech., 68, 1839-1887, doi:10.1016/S0013-7944(01)00037-6.

Schulson, E. M. (2004), Compressive shear faults within arctic sea ice: Fracture on scales large and small, J. Geophys. Res., 109, C07016, doi:10.1029/2003JC002108

Schulson, E. M., A. L. Fortt, D. Iliescu, and C. E. Renshaw (2006), On the role of frictional sliding in the compressive fracture of ice and granite: Terminal vs. post-terminal failure, Acta Mater., 54, 3923-3932, doi:10.1016/j.actamat.2006.04.024.

Tremblay, L. B., and L. A. Mysak (1997), Modeling sea ice as a granular material, including the dilatancy effect, J. Phys. Oceanogr., 27, 2342-2360

Weiss, J., and E. M. Schulson (2007), Sea ice rheology from in-situ, sattelite and laboratory observations: Fracture and friction, Earth Planet. Sci. Lett., 255, 1-8, doi:10.016/j.eps1.2006.1.033.

Weiss, J., and E. M. Schulson (2009), Coulombic faulting from the grain scale to the geophysical scale: Lessons from ice, J. Phys. D: Appl. Phys., 42, 214017, doi:10.1088/0022-3727/42/21/214017.
Wilchinsky, A. V., and D. L. Feltham (2004), A continuum anisotropic model of sea-ice dynamics, Proc. R. Soc. A, 460, 2105-2140, doi:10.1098/rspa.2004.1282.

Wilchinsky, A. V., and D. L. Feltham (2006a), Modelling the rheology of sea ice as a collection of diamond-shaped floes, J. Non-Newtonian Fluid Mech., 138, 22-32, doi:10.1016/j.jnnfm.2006.05.001.

Wilchinsky, A. V., and D. L. Feltham (2006b), Anisotropic model for granulated sea ice dynamics, J. Mech. Phys. Solids, 54, 1147-1185, doi:10.1016/j.jmps.2005.12.006.

Wilchinsky, A. V., D. L. Feltham, and P. Miller (2006), A multi-layer sea ice model accounting for sliding friction, J. Phys. Oceanogr., 36, 1719-1738.

Wilchinsky, A. V., D. L. Feltham, and M. A. Hopkins (2010), The effect of shear rupture on aggregate scale formation in sea ice, J. Geophys. Res., 115, C10002, doi:10.1029/2009JC006043.

Wilchinsky, A. V., D. L. Feltham, and M. A. Hopkins (2011), Modelling the reorientation of sea-ce faults as the wind changes direction, Ann. Glaciol., 52, 83-90.

D. L. Feltham and A. V. Wilchinsky, National Centre for Earth Observation, Centre for Polar Observation and Modeling, University College London, Gower Street, London WC1E 6BT, UK. (dlf@cpom. ucl.ac.uk; aw@cpom.ucl.ac.uk) 